Arnulf Deppermann (Mannheim)

\title{
Einleitung: Das Deutsch der Migranten
}

„Das Deutsch der Migranten“ - ein Titel, der schön griffig ist, der neugierig macht und provoziert. Ein Titel, der darauf verweist, dass soziale Prozesse unmittelbar Entwicklungen und Veränderungen von Sprache und Kommunikation in unserer Gesellschaft nach sich ziehen. Natürlich: Die Einfachheit des Titels ist tückisch, ja vielleicht gar irreführend. Denn es gibt weder „die Migranten“ noch „ein Deutsch“, das sie alle sprechen. Ethnische Herkunft, Nationalität, kulturelle Orientierung und sprachlicher Habitus werden allzu oft in eins gesetzt, als ergäbe sich aus dem einen zwangsläufig auch das andere. Doch die erste Lehre einer Soziolinguistik der Globalisierung (Blommaert 2010) ist, dass auch sprachlich und gerade in sprachlichkommunikativer Hinsicht in unserer Gesellschaft „Superdiversität“ (Vertovec 2007) schon längst nicht mehr die Ausnahme, sondern zunehmend die Regel ist (Blommaert/Rampton 2011). Wenn wir vom „Deutsch der Migranten" sprechen, dann sprechen wir vom türkischstämmigen Bundestagsabgeordneten dritter Generation wie von der frisch zugezogenen Heiratsmigrantin, von der Japanerin, die für ein paar Monate in der deutschen Filiale ihrer Firma arbeitet, wie vom Roma, der den Handel mit Autos zwischen Deutschland und Rumänien organisiert. So sehr also „Das Deutsch der Migranten“ sofort das Stereotyp des bildungsfernen, männlichen, vielleicht gar in einer Parallelgesellschaft lebenden Migranten aus Süd(ost)europa aufzurufen scheint, so sehr verweist es bei genauerem Hinsehen gerade auf keinen einheitlichen Gegenstand, sondern auf die Dynamik kultureller und sprachlicher Vielfalt, die sich in unserer Gesellschaft gegenwärtig entwickelt - mit all ihren Potenzialen und Problemen und den sich daraus ergebenden Herausforderungen für die linguistische Forschung, nicht nur in der Germanistik. Allein schon die Tatsache, dass das Medieninteresse an wohl keiner der bisherigen Jahrestagungen des IDS so groß war wie an dieser, und die nahezu 500 TeilnehmerInnen zeigen, wie sehr die mit dem Thema angesprochenen Fragen und Entwicklungen Wissenschaft und Öffentlichkeit beschäftigen.

Migration erzeugt eine Vielfalt neuer Konstellationen sprachlicher, kommunikativer und sozialer Art. Eine erste Lehre ist es, diese Vielfalt nicht über einen Kamm zu scheren. Die manchmal sehr holzschnittartige Diskussion („Deutschland schafft sich ab“, Sarrazin 2010) zu versachlichen und zu differenzieren war also ein Ziel dieser Tagung, das heißt: Zunächst einmal den Blick auf die Fakten der sprachlichen Kommunikation zu richten. Um vereinfachende Problemdiagnosen und ebensolche Lösungen, die wir 
oft genug im gesellschaftlichen Diskurs zum Thema „Sprache“ hören, zu begegnen, sind gerade auch die Germanistik und andere Sprachwissenschaften gefragt.

Die Beiträge der Tagung und also auch dieses Buchs gliedern sich in vier Stränge:

- Migrationsbedingte Sprechweisen,

- Kommunikation zwischen Einheimischen und Zuwanderern,

- Sprachbiographien von Migranten,

- Erwerb des Deutschen als Zweitsprache.

Die Frage, die in der Öffentlichkeit wohl am Hitzigsten diskutiert wird, wenn es um das Thema „Sprache und Migration“ geht, ist die nach der Eigenart migrationsbedingter Sprechweisen. Gibt es umschriebene Ethnolekte des Deutschen? Wo kommen sie her? Wie sehen ihre Strukturen aus? Was haben sie mit den Erstsprachen der Sprecher zu tun? Im Brennpunkt steht dabei besonders die Frage: Handelt es sich bei diesen Sprechweisen um einen Habitus, der auf ein begrenztes sprachliches Repertoire verweist? Oder sind es frei gewählte Stile, die flexibel je nach Gesprächssituation und Kommunikationszweck benutzt werden?

Die Untersuchungen in diesem Band von Peter Auer und Heike Wiese zeigen, dass wir es mit mehr als individuellen Genus- oder Kasusfehlern zu tun haben. Wir sehen Besonderheiten der Wortstellung und der Rhythmisierung,

Gestern (.) hab ich angefangen (.) Training.

der Nichtrealisierung von Artikeln und Präpositionen

Muss $=$ sch morgen Schule gehen.

oder bestimmter Konstruktionen für bestimmte Handlungen wie Bewertungen

Gestern das war voll der coole Film.

Inwieweit diese Sprechweisen auf dem Weg sind, sich zu stabilen, tradierten Varietäten auszubilden, welche Formen in der Sprachbiographie der Sprecher auf Dauer erhalten bleiben und was schließlich auch in andere Varietäten (wie Spielarten der Jugendsprache) und schließlich in die Gemeinsprache übergehen wird, ist noch offen. Hier ist der Blick auf andere Länder wie Großbritannien, die aufgrund ihrer kolonialen Vergangenheit schon länger Erfahrungen mit der Immigration exolingualer Sprechergruppen haben, aufschlussreich. Ben Ramptons Beitrag zeigt, wie sich aus ursprünglich jugendprachlichen, ethnisch markierten „Ghetto“-Sprechweisen in London urbane Sprechweisen herausbilden, die sprachliche Formen unterschied- 
lichster Variationsquellen synthetisieren und flexibel, je nach Adressat und Kontext kombinieren. Migranten kommen aber nicht nur mit nicht-deutschen, sondern auch mit deutschen Herkunftssprachen in unser Land. So zum Beispiel die Russlanddeutschen, zumindest die, die vor der Jahrtausendwende einwanderten. Was wird aus dem von ihnen mitgebrachten Deutsch? Wie passt es sich den regionalen und überregionalen Varietäten an, die im Aufnahmeland gesprochen werden? Welche Merkmale sind besonders widerständig? Nina Berends Längsschnittuntersuchung zur sprachlichen Anpassung russlanddeutscher Zuwanderer gibt Antworten. Fragen nach dem Deutsch der Migranten sind also immer auch Fragen nach der inneren Mehrsprachigkeit des Deutschen. Aber Migration führt natürlich auch zu mehrsprachigen Praktiken, die das Deutsche und die Herkunftssprachen umfassen. Ibrahim Cindark zeigt, dass der Wechsel zwischen Sprachen je nach sozialer Gruppe sehr unterschiedlich praktiziert und bewertet wird und somit auch einen Blick auf Spracheinstellungen und Identitätsentwürfe gibt. Früher wurde Mehrsprachigkeit unter Stichworten wie „Semilingualismus" vor allem als Problem gesehen. Heute setzt sich immer mehr die Erkenntnis durch, welchen Reichtum sie identitätsbezogen, kulturell und auch für das berufliche Fortkommen eröffnen kann.

Differenzen und Hürden in der Kommunikation zwischen Einheimischen und Zuwanderern sind aber nicht nur und oft gar nicht in erster Linie begrenzten Sprachkompetenzen geschuldet. Das zeigt sich besonders in der Kommunikation mit Institutionen. Fremdheit ist nicht etwas bloß Faktisches, sie ist selbst eine Orientierungsgröße, die schnell für alle Beteiligten in oft problematischer Weise leitend wird. Ulrich Reitemeier zeigt, wie die wechselseitige Orientierung an kultureller Differenz und damit an einer Differenzidentität zur Zuschreibung inakzeptabler Motive und zu eskalierenden Gesprächsprozessen, in denen der Partner letzten Endes als irrational und moralisch suspekt abgestempelt wird, führt. Uta Quasthoff und Ludger Hoffmann diskutieren Hürden in der Kommunikation mit der Verwaltung und ihre Deutung und Verarbeitung durch die betroffenen Migranten, die von Anpassung über die Entwicklung individueller Erfolgsstrategien bis hin zur Selbstwahrnehmung als Opfer reichen.

Erfahrungen mit individueller und gesellschaftlicher Mehrsprachigkeit, mit multilingualer und interkultureller Kommunikation, mit Sprachbewertungen und Fremdbildern verdichten sich zu Sprachbiographien. Die Beiträge von Katharina Brizić und Patrick Stevenson zeigen, dass es sehr oft dabei um mehr als nur die Herkunftssprache und die Sprache des Aufnahmelandes geht: Zahlreiche Sprachen können im Lauf des Lebens in unterschiedlichen sozialen Kontexten (privat, schulisch, beruflich), an verschiedenen Orten und in unterschiedlichen biographischen Phasen relevant werden, Drittsprachen erlangen Bedeutung in Beziehungen von Partnern mit unterschiedlichen Herkunftssprachen, stigmatisierte Sprachen werden 
in der Fremde zugunsten anderer Sprachen, die nicht die des Aufnahmelandes sind, abgelegt. Der Blick auf die sprachbiographischen Erfahrungen von Menschen, die selbst als Deutschsprachige zur Migration und damit auch in den Weg in eine fremde Sprache gezwungen wurden, mag viele vielleicht besser die tiefgreifenden Infragestellungen des Selbstverständnisses und der sprachlich-kommunikativen Selbstverständlichkeiten nachvollziehen lassen, die solche biographischen Umbrüche mit sich bringen können. Anne Bettens Studie über die Jeckes, die nach Israel vor dem Holocaust ausgewanderten deutschen Juden, legt Zeugnis über Zusammenhänge zwischen Sprachbewertung, ideologisch-kultureller Orientierung und Sprachloyalität in der neuen Umgebung und ihrer Bedeutung für das Selbstverständnis der Sprecher ab.

Wenn vom „Deutsch der Migranten“ die Rede ist, dann geht es auch darum, wie der Zweitspracherwerb verläuft und wie er pädagogisch zu unterstützen ist. Verläuft er anders als der Erstspracherwerb? Wie hängt er vom Erwerbsalter und den Strukturen der Erstsprache ab? Welche Erwerbsaufgaben stellen besondere Hürden dar? Petra Schulz befasst sich mit diesen Fragen in Bezug auf den Erwerb des Verständnisses von $w$-Fragesätzen, Ulrich Mehlem blickt auf die Entwicklung von mündlichen und schriftlichen Erzählkompetenzen. Linguistisch gesicherte Erkenntnisse sind hier vonnöten, denn nur mit ihnen ist es möglich, Sprachdidaktik und Sprachförderung effektiv zu gestalten. Ursula Bredel zeigt dies für den Orthographieerwerb und diskutiert, wie die Orthographiedidaktik zu optimieren ist, um Lerner besser zu unterstützen.

Die Dokumentation der Podiumsdiskussion zum Tagungsthema vervollständigt diesen Band. In ihr werden die hier besprochenen Aspekte aufgegriffen und durch weitere vervollständigt, wie die Frage nach der Rolle der Wissenschaft in der öffentlichen Diskussion, die Legitimität der wissenschaftlichen Diskurse über „Migrantendeutsch“ und „Ethnolekte“, den Zusammenhang von Bildungserfolg und sprachlichen Kompetenzen oder die Notwendigkeit und die Ausgestaltung von Sprachtests.

Der Erfolg der Tagung und das breite Interesse, auf das die verschiedenen Facetten des Tagungsthemas gestoßen sind, zeigen, dass wir es mit einem der Bereiche der gegenwärtigen Linguistik zu tun haben, in denen in den kommenden Jahren weitere neue Entwicklungen zu erwarten sind. Die Fragen der Ausbildung neuer Varietäten und ihrer linguistischen Strukturen, ihres Einflusses auf den Sprachwandel, die Entwicklung gesellschaftlicher und individueller Mehrsprachigkeit und die Entstehung neuer kommunikativer Interkulturen in Organisationen und im Privatleben, die Erforschung multilingualer Sprachbiographien und des Zweitspracherwerbs sowie die Entwicklung von linguistisch fundierten pädagogischen Angeboten und ihre feste Verankerung in Krippen, Kindergärten und Schulen sind Herausforderungen, die der linguistischen Forschung in den kommenden 
Jahren genügend Stoff geben werden. Dass wir auf der Jahrestagung des IDS Beispiele prominenter Forschung zu all diesen aktuellen Entwicklungen präsentieren konnten, ist auch das Verdienst von Nina Berend, Inken Keim, Angelika Redder und Rosemarie Tracy, die mich im wissenschaftlichen Organisationskommitee dieser Tagung mit ihrer Expertise und ihren Kontakten unterstützt haben. Ihnen gilt mein Dank, ganz wesentlich zum Zustandekommen einer vielperspektivischen Tagung auf ein gesellschaftlich brennendes wie grundlagenwissenschaftlich herausforderndes Thema beigetragen zu haben.

\section{Literatur}

Blommaert, Jan (2010): The sociolinguistics of globalization. Cambridge.

Blommaert, Jan/Rampton, Ben (2011): Language and Superdiversity. In: Diversities 13, 2, S. 1-22.

Sarrazin, Thilo (2010): Deutschland schafft sich ab. Wie wir unser Land aufs Spiel setzen. München.

Vertovec, Steven (2007): Super-diversity and its implications. In: Ethnic and Racial Studies 29, 6, S. 1024-54. 